\title{
Domains of Research Ethics
}

\author{
P.Subharani \\ Associate Professor, Saveetha College of Nursing, Saveetha University, India
}

\section{Introduction -Domains of Research Ethics}

Ethics is rooted in the ancient Greek philosophical inquiry of moral life. It refers to a system of principles which can critically change previous considerations about choices and actions. IIt is said that ethics is the branch of philosophy which deals with the dynamics of decision making concerning what is right and wrong. Scientific research work, as all human activities, is governed by individual, community and social values. Research ethics involve requirements on daily work, the protection of dignity of subjects and the publication of the information in the research.

Key words -Ethics, Information, Intellectual Property

However, when nurses participate in research they have to cope with three value systems; society; nursing and science. The societal values about human rights, the nursing culture based on the ethic of caring and the researcher's values about scientific inquiry[1.]

Since 2001, the UK Government health departments have implemented the Research Governance Frame work to strengthen public confidence in research and improve the management and monitoring of research. The framework relates to set standards that outline the key principles of a quality research culture in five governance domains.

Research adheres to its key principles. The research governance standards relate to five domains: Ethics; Science;Information; Health, Safety And Employment; Finance And Intellectual Property.

The Ethic domain is concerned with ensuring the dignity, rights, safety and well-being of participants who are the primary consideration in any research study. In addition, data protection, ethics committees, informed consent and confidentiality are integral concerns to the research process.

The Science domain argues that unnecessary research duplication is unethical and that only original high quality research should be generated. In practice, this means that existing sources of evidence should be used and all research proposals should be subject to peer review.

Special guidance is given for research involving human embryos, animals, genetically modified organisms, and medicines.

The Information domain highlights the need for information on research and subsequent findings should be accessible to the public through publication.

The Health, Safety and Employment domain recommends that the safety of research participants and staff is assured by adhering to health and safety regulations. New or existing medical devices need to be approved by the medical devices agency to ensure safety for staff and patients.

Finally, the Finance and Intellectual property domain advocates compliance with the law and rules set for the use of public funds. Compensation is recommended for anyone harmed as a result of studies.

Intellectual property (IP) is concerned with inventions, know-how (knowledge), copyrights and database rights, designs, trademarks and materials..[2]

For example, it should be agreed who will be credited with funds and authorship at a study's outset. 


\section{Conclusion}

Researchers should inculcate the basic qualities like facts finding, critical analysis, and scientific enquiry for providing the quality studies.

\section{Reference}

[1] Johnstone M. Bioethics. A Nursing Perspective, 5th edition Churchill Livingstone Elsevier, 2009. Georgia Fouka Marianna Mantzorou RN, PhD.

[2] Key information

Visit the Department of Health's website:

www.dh.gov.uk.

For the UKCRC,

visitwww.ukcrc-rgadvice.org.

ROYAL COLLEGE OF NURSING 